\title{
A Coarse-to-Fine Strategy for Vehicle Motion Trajectory Clustering
}

\author{
Xi Li, Weiming Hu, Wei Hu \\ National Laboratory of Pattern Recognition, Institute of Automation, CAS, Beijing, China \\ Email: \{lixi,wmhu,whu\}@nlpr.ia.ac.cn
}

\begin{abstract}
High-level semantic understanding of vehicle motion behaviors is often based on vehicle motion trajectory clustering. In this paper, we propose an effective trajectory clustering framework in which a coarse-to-fine strategy is taken. Our framework consists of four stages: trajectory smoothing, feature extraction, trajectory coarse clustering and trajectory fine clustering. Wavelet decomposition is imposed on raw trajectories to reduce noise in the trajectory smoothing stage. Besides the commonly used positional feature, a novel feature called trajectory directional histogram is proposed to describe the statistic directional distribution of a trajectory in the feature extraction stage. Both coarse clustering and fine clustering are based on a novel graphtheoretic clustering algorithm called dominant-set clustering, but they deal with different trajectory features. Experiments in our pre-labeled trajectory database demonstrate that the proposed trajectory clustering framework possesses a very high accuracy.
\end{abstract}

\section{Introduction}

In recent years, some researches have been done in the field of learning the distribution of motion trajectories. In [1], two competitive neural networks connected by a leaky neuron layer are used to model the probabilistic distribution of flow vectors and trajectories. Compared with [1], fuzzy self-organizing map (FSOM) proposed in [2] has a much simpler architecture because it takes the whole trajectory rather than discrete flow vectors as input. But it still takes a very long time for training due to the high computational complexity of SOM. More recent work on trajectory modeling clusters trajectories directly compared with early work. Graph cut is used for trajectory clustering in [3], where Hausdorff distance is exploited to measure the distance between any two trajectories. Spectral clustering outperforms graph cut in the case of multi-class trajectory clustering in [4], but the clustering results are sensitive to trajectory lengths because of using Euclidean distance as the distance measure. The common ground of the above trajectory clustering approaches is that they all ignore directional distribution information. Directional distribution information describes the rough "trend" of a trajectory. It is less sensitive to trajectory length and thus is an effective and efficient feature for trajectory clustering. In our work, a novel directional feature called trajectory directional histogram (TDH) is proposed to describe the statistic directional distribution of one trajectory. Moreover, we define a "coarse cluster" as a set of trajectories sharing with similar trends and being easily extracted and separated from other trajectories using TDH.

In our trajectory clustering framework, we take advantage of a newly proposed graph-theoretic clustering method called dominant-set clustering [5]. It takes on an iterative bipartition manner and split a "dominant set" from a weighted graph in each round. With flexibility in determining the number of clusters and low computational complexity, it is very suitable for large-scale trajectory clustering.

The rest of this paper is organized as follows: In Section 2, we describe our trajectory clustering framework including overview of our clustering framework, trajectory smoothing, feature extraction, dominant-set clustering, trajectory coarse clustering and trajectory fine clustering. Experimental results are shown in Section 3. In Section 4, we conclude our work.

\section{Our framework for trajectory clustering}

\subsection{Overview of our framework}

Our trajectory clustering framework includes four stages: 1)trajectory smoothing; 2)feature extraction; 3)trajectory coarse clustering; 4)trajectory fine clustering. In the trajectory smoothing stage, two-layer wavelet decomposition is used to smooth the raw trajectories. In the feature extraction stage, two kinds of features are extracted. One is trajectory resampling point set (TRPS), and the other is a novel feature called trajectory directional histogram (TDH). TRPS contains the positional information of one trajectory while TDH describes its statistic directional distribution. In the coarse clustering stage, TDH is used to 
obtain coarse trajectory clusters, where trajectories share similar "trends". In the fine clustering stage, trajectories in each of those coarse clusters are further clustered into fine clusters using TRPS. By this way, different features complementary with each other are dealt with at different levels to improve the whole efficiency and accuracy of our framework.

\subsection{Trajectory smoothing}

In this paper, multi-resolution wavelet decomposition [6] is used to smooth trajectories. As we all know, any squareintegrable signal can be decomposed into a high-frequency signal and a low-frequency signal by wavelet decomposition. The high-frequency signal provides detailed information of the original signal while the low-frequency signal reserves the main structural characteristics. Consequently, the low-frequency signal can be viewed as a "smoothing" one of the original signal.

A raw trajectory can be represented as follows:

$$
\left(\begin{array}{l}
X \\
Y
\end{array}\right)=\left(\begin{array}{llll}
x_{1} & x_{2} & \cdots & x_{n} \\
y_{1} & y_{2} & \cdots & y_{n}
\end{array}\right)
$$

where $n$ is the number of points on that trajectory. We impose a two-layer wavelet decomposition respectively on $X$ and $Y$ and get the position expression of the smoothed trajectory:

$$
\left(\begin{array}{l}
X^{s} \\
Y^{s}
\end{array}\right)=\left(\begin{array}{llll}
x_{1}^{s} & x_{2}^{s} & \cdots & x_{n}^{s} \\
y_{1}^{s} & y_{2}^{s} & \cdots & y_{n}^{s}
\end{array}\right)
$$

where $X^{s}$ and $Y^{s}$ respectively denotes the smoothing signals of the original ones $X$ and $Y$ at the resolution $2^{-2}$.

One of the raw trajectories in our experiments and its smoothed trajectory are respectively shown in Figure 1(a) and Figure 1(b). We can see that although the trajectory gets smoothed, its shape or intrinsic structural relationship keeps unchanged.

\subsection{Feature extraction}

In this stage, we will extract TRPSs and TDHs of trajectories. The details are described below.

First, we extract TRPSs of trajectories. All smoothed trajectories are resampled at the same space interval by linear interpolation. As a result, they all have the same number of points. We use $r$ to denote the number of points of each trajectory after resampling. In this case, we obtain TRPS of a trajectory:

$$
\text { TRPS }=\left\{x_{1}, x_{2}, \ldots, x_{r}, y_{1}, y_{2}, \ldots, y_{r}\right\}
$$

And then we extract TDHs of trajectories. Any trajectory after smoothing and resampling can be represented by a set Q:

$$
\left\{\begin{array}{l}
Q=\left\{f_{1}, f_{2}, \ldots, f_{j}, \ldots, f_{r-2}, f_{r-1}\right\} \\
f_{j}=\left(x_{j}, y_{j}, d x_{j}, d y_{j}\right) \\
d x_{j}=x_{j+1}-x_{j} \\
d y_{j}=y_{j+1}-y_{j}
\end{array}\right.
$$
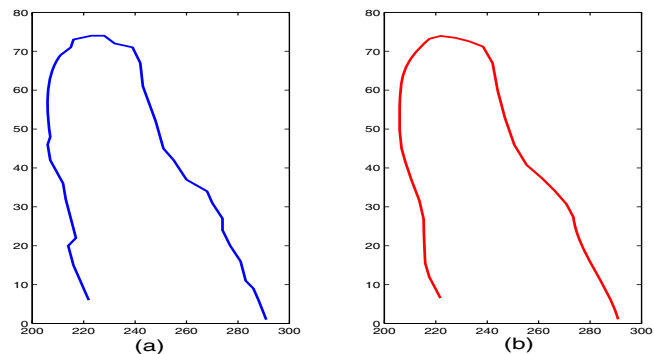

\section{Figure 1. (a) An original trajectory (b) The smoothed trajectory}

where $j \in\{1,2, \ldots, r-2, r-1\}$. The directional angle $\theta_{j}$ of the $j$ th point in the trajectory is defined as follows:

$$
\theta_{j}=\left\{\begin{array}{l}
\arctan \left(\frac{d y_{j}}{d x_{j}}\right), d x_{j}>0 \\
\arctan \left(\frac{d y_{j}}{d x_{j}}\right)+\pi, d x_{j} \leq 0, d y_{j} \geq 0 \\
\arctan \left(\frac{d y_{j}}{d x_{j}}\right)-\pi, d x_{j} \leq 0, d y_{j}<0
\end{array}\right.
$$

where $\left(d x_{j}\right)^{2}+\left(d y_{j}\right)^{2} \neq 0$ and $\theta_{j} \in[-\pi,+\pi)$. And then we divide the directional interval $[-\pi, \pi)$ into $N$ equal subintervals, which are $I_{1}, I_{2}, \ldots, I_{N}$. The length of each subinterval is $\triangle \alpha(\triangle \alpha=2 \pi / N) . \theta_{j}$ belongs to $I_{i}$ if $(i-1) \triangle \alpha \leq$ $\theta_{j}<i \triangle \alpha$, where $1 \leq i \leq N$. In this way, all points in the trajectory are mapped into their corresponding directional subintervals. And then we calculate $\frac{M_{i}}{M}$, where $M_{i}$ denotes the number of points in $I_{i}$ and $M$ denotes the total number of points in all directional subintervals. As a result, we get a directional distribution which can be viewed as a normalized histogram. $N$ bins of the histogram correspond to $N$ directional subintervals. The histogram describes statistical directional characteristics of the trajectory. Consequently, we call it TDH, which is a novel feature to represent a trajectory. TDH of a trajectory is represented as:

where $p_{i}=\frac{M_{i}}{M}$.

\subsection{Dominant-set clustering}

Dominant-set clustering is a new graph-theoretic clustering algorithm proposed by Pavan et al. in [5]. It introduces a novel notion which is a "dominant set" defined as follows:

A non-empty subset $X$ of vertices $X \subseteq V$, such that $W(K)>0$ for any non-empty $K \subseteq X$, we say $X$ is a "dominant set" if $w_{X}(i)>0$ for all $i \in X$ and $w_{X \cup\{i\}}(i)<0$ for all $i \notin X$, where $W(K)$ denotes the total weight of the vertex set $K$ and $w_{X}(i)$ denotes a measure of the overall similarity between vertex $i$ and the vertices of $X$ with respect to the overall similarity among the vertices in $X$.

Meanwhile, it has been proved in [5] that finding a "dominant set" is equivalent to solving an quadratic program:

$$
\begin{array}{ll}
\operatorname{maximize} & \frac{1}{2} x^{T} A x \\
\text { subject to } & x \in \Delta
\end{array}
$$

where $A$ is the graph's adjacency similarity matrix and $\triangle=$ $\left\{x \in R: x \geq 0\right.$ and $\left.\sum_{i=1}^{n} x_{i}=1\right\}$. 
Table 1. Dominant-set clustering algorithm

Given: A data set $X=\left\{x_{1}, x_{2}, \ldots, x_{L}\right\}$

1. Create a graph $G=(V, E)$, where $V=\{1, \ldots, L\}$ is the vertex set and $E \subseteq V \times V$ represents the edge set. $w_{i j}$ denotes the edge-weight between sample $i$ and sample $j$, where $w_{i j}=\exp \left(\operatorname{dist}\left(x_{i}, x_{j}\right) / 2 \sigma^{2}\right)$. $\operatorname{dist}(\cdot, \cdot)$ denotes a kind of distance. And then $G$ is represented as an symmetric matrix $A=$ $\left(a_{i j}\right)$,which is defined as:

$$
a_{i j}= \begin{cases}w_{i j} & \text { if }(i, j) \in E \\ 0 & \text { otherwise }\end{cases}
$$

2. Solve the quadratic program (7), which is solved by replicator equation: $x_{i}(t+1)=x_{i}(t) \frac{(A \mathbf{x}(t))_{i}}{\mathbf{x}(t)^{T} A \mathbf{x}(t)}$. A strict local solution is represented as $x^{*}$. Let $\Omega_{1}$ denote the vertex set $\left\{i:\left(x^{*}\right)_{i}>0\right\}$ and $\Omega_{2}$ denote the vertex set $V \backslash \Omega_{1}$.

3. Two sub-graphs $G_{1}=\left(\Omega_{1}, E_{1}\right)$ and $G_{2}=\left(\Omega_{2}, E_{2}\right)$ are obtained. $G_{1}$ corresponds to a "dominant set".

The specific steps of dominant-set clustering are detailed in Table 1 .

\subsection{Trajectory coarse clustering}

In this stage, we take a divisive hierarchical clustering structure for trajectory clustering. The entire data set is first considered to be one cluster and is then broken down into smaller and smaller sub-clusters by dominant set clustering until the intra-cluster tightness of each sub-cluster reach some criterion. Results of hierarchical clustering can be represented as a binary tree. Its root represents the entire data set. Each interior node of the tree represents a temporary cluster needing partitioning. Each terminal node of the tree represents a final cluster, where trajectories have homogeneous "trends". We call it a "coarse cluster", which needs further clustering in the fine clustering stage described in Section 2.6. The feature used for coarse clustering is TDH, and the Bhattacharyya distance is used as the distance measure between two trajectories.

The specific steps of coarse clustering are shown in Figure 2(a), where $T^{H}$ is a threshold and $J^{H}(c)$ is a criterion function used for evaluating the tightness of a cluster $c$. $J^{H}(c)$ is defined as:

$$
J^{H}(c)=\max _{i, j \in c} H(i, j)
$$

where $H(i, j)$ denotes the Bhattacharyya distance between the $i$ th trajectory and the $j$ th trajectory:

$$
H(i, j)=\left[1-\sum_{b=1}^{N} \sqrt{\mathrm{TDH}_{i b} \mathrm{TDH}_{j b}}\right]^{1 / 2} \in[0,1]
$$

where $\mathrm{TDH}_{i b}$ denotes the $b$ th element of the $i$ th trajectory's corresponding $\mathrm{TDH}$ and $\mathrm{TDH}_{j b}$ denotes the $b$ th element of the $j$ th trajectory's corresponding TDH.

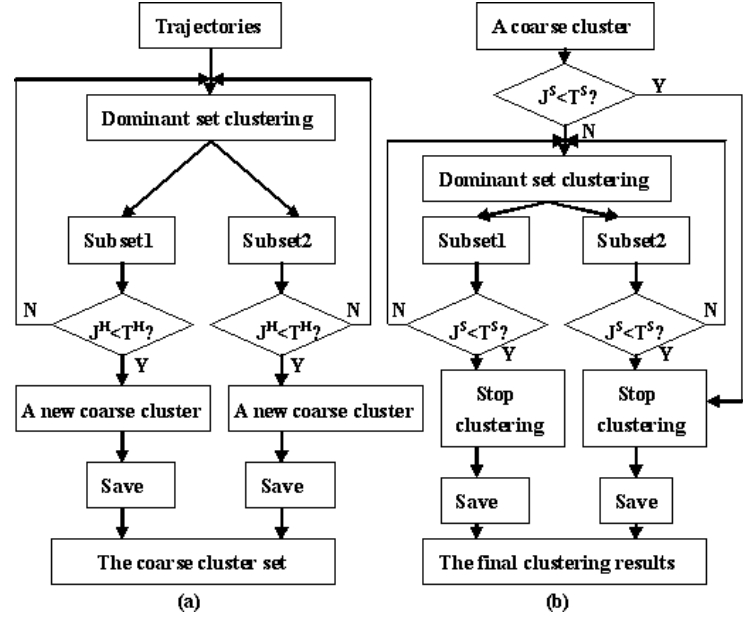

Figure 2. (a)Coarse clustering framework (b)Fine clustering framework

\subsection{Trajectory fine clustering}

In Section 2.5 , a set of "coarse clusters" are obtained. Trajectories in each "coarse cluster" have similar directional characteristics, but they have different positional characteristics. So it is necessary for trajectories to be clustered further. The feature used for fine clustering is TRPS, and the Euclidean distance is used as the distance measure between two trajectories.

The structure of fine clustering is very similar to that of coarse clustering. The divisive hierarchical clustering structure is also taken. There are also a threshold $T^{S}$ and a criterion function $J^{S}(c)$ used for evaluating the tightness of a cluster $c . J^{S}(c)$ is defined as follows:

$$
J^{S}(c)=\max _{i, j \in c} S(i, j)
$$

where $S(i, j)$ denotes the Euclidean distance between trajectory $i$ and trajectory $j$ :

$$
S(i, j)=\left\|\operatorname{TRPS}_{i}-\operatorname{TRPS}_{j}\right\|_{2}
$$

where TRPS $_{i}$ denotes the $i$ th trajectory's corresponding TRPS, TRPS ${ }_{j}$ denotes the $j$ th trajectory's corresponding TRPS and $\|\cdot\|_{2}$ denotes $L_{2}$ norm. The specific steps of fine clustering are shown Figure 2(b).

\section{Experimental results}

In our experiments, a total number of 1200 trajectories represented by yellow lines in Figure 3(a) are clustered. They are pre-labeled into fifteen clusters manually in order to test the performance of our framework efficiently. In the feature extraction stage, we set $N=24$ and $r=20$. In the trajectory coarse clustering stage, we set the threshold $T^{H}=0.5$ and the scaling parameter $\sigma=0.7$ of dominantset clustering, which are obtained from experiments. Raw trajectories are clustered into nine "coarse clusters". In each "coarse cluster", trajectories have very similar directions. We just choose to display seven dominant "coarse clusters" 


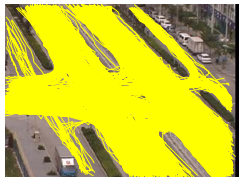

(a)

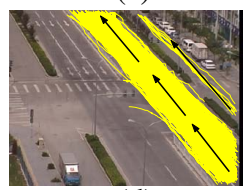

(d)

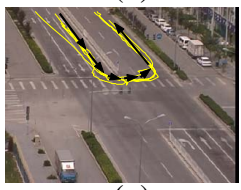

(g)

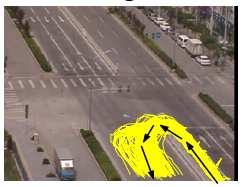

(j)

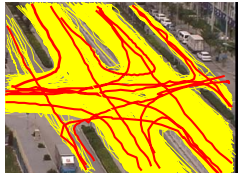

(b)

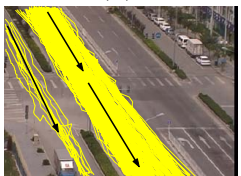

(e)

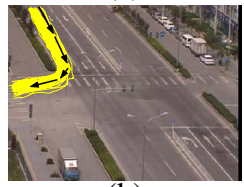

(h)

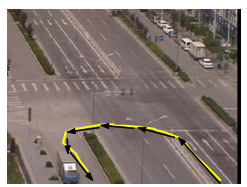

(k)

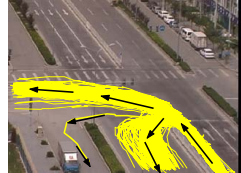

(c)

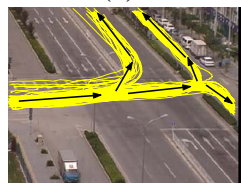

(f)

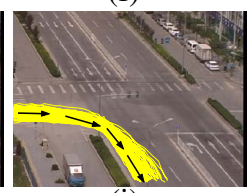

(i)

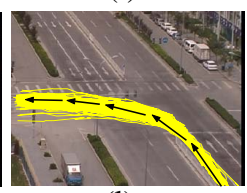

(1)
Figure 3. (a) Raw trajectories. (b) Final clustering results. (c)-(i) Seven coarse clusters. (j)-(I) Fine clustering results of Figure (c).

for better visualization. They are shown in Figure 3(c)(i), where black arrows represent the directions of trajectories. The other two "coarse clusters" are treated as outliers. In the trajectory fine clustering stage, we set the threshold $T^{S}=30$ and the scaling parameter $\sigma=70$ of dominant-set clustering, which are also from experiments. In this stage, trajectories are clustered further by their positional features. We take Figure (c) for example to illustrate the process of fine clustering. In Figure (c), we see there are obviously three clusters of trajectories which all head left. But the three clusters of trajectories have different positional features. Therefore, we cluster them further according to their positional features. The clustering results after fine clustering are shown in Figure 3(j)-(1). At last, fifteen final clusters are obtained in this stage. The number of final clusters is in accordance with that of clusters pre-labeled manually. In order to represent trajectory clusters efficiently, we introduce the template trajectory which is the one with minimum distances sum of distances to the other trajectories in the same cluster. The final clustering results are displayed in Figure 3(b), where fifteen template trajectories represented by red thick lines correspond to fifteen clusters of trajectories.

In order to evaluate the performance of our trajectory clustering framework quantitatively, we calculate the clustering accuracy of each cluster. We use Figure 4 to show the clustering accuracy of our framework. From Figure 4, we can see the performance of our framework is good.

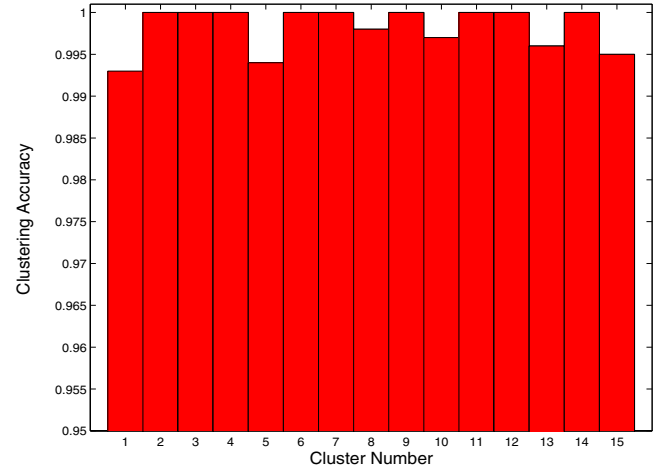

Figure 4. Clustering accuracy of our framework.

\section{Conclusion}

We have proposed a hierarchical trajectory clustering framework based on dominant-set clustering. Wavelet decomposition has been used for smoothing trajectories. Meanwhile, a novel feature called trajectory directional histogram describing the directional distribution information of a trajectory has been proposed. Original pre-labeled trajectories are clustered in our trajectory clustering framework. Experimental results demonstrate the effectiveness of our framework. Vehicle motion behavior analysis is our future work.

\section{Acknowledgment}

This work is partly supported by NSFC (Grant No. 60520120099) and Natural Science Foundation of Beijing (Grant No. 4041004).

\section{References}

[1] N. Johnson and D. Hogg, "Learning the Distribution of Object Trajectories for Event Recognition", British Machine Vision Conference, 1995.

[2] Weiming Hu, Dan Xie, Tieniu Tan, Steve Maybank, "Learning Activity Patterns Using Fuzzy SelfOrganizing Neural Network", IEEE Tans. Systems, Man and Cybernetics-Part B, 34(3):1618-1626,2004.

[3] Imran N.Junejo, Omar Javed, Mubarak Shah, "Multi Feature Path Modelling for Video Surveillance", Int. Conf. Pattern Reocognition,Vol.2, pp.716-719, 2004.

[4] Zhouyu Fu, Weiming Hu, Tieniu Tan, "Similarity Based Vehicle Trajectory Clustering and Anomaly Detection”, Int. Conf. on Image Processing,Vol.2, pp.602-605, 2005.

[5] Massimiliano Pavan and Marcello Pelillo, "A New Graph-Theoretic Approach to Clustering and Segmentation", In Proc. CVPR'03, Vol. 1, pp. I-145-I-152, 2003.

[6] Stephane G. Mallat, "A Theory for Multiresolution Signal Decomposition: The Wavelet Representation”, IEEE Trans. PAMI, 11(7):674-693, July 1989. 